\section{Bienvenida: X Simposio Internacional de la Sociedad Colombiana de Cardiología y cirugía Cardivascular, Simposio Internacional de Electrofisiología y I Congreso Colombiano de Cardiología para Médicos de Atención Primaria}

\section{Welcome to the 10th International Symposium of the Colombian Society of Cardiology and Cardiovascular Surgery, International Symposium of Electrophysiology, and the $1 \mathrm{st}$ Colombian Congress of Cardiology for Primary Care Physicians}

\section{Estimados Colegas y Amigos:}

Reciban un cordial saludo en nombre de la Junta Directiva 2016-2018 de la Sociedad Colombiana de Cardiología y Cirugía Cardiovascular y el mío.

Bien saben todos que las enfermedades cardio-cerebroreno-angio-vasculares (comúnmente conocidas como cardiovasculares), ocupan la primera causa de morbi-mortalidad y comorbilidad en el mundo occidental y en casi todo el planeta, y que somos precisamente los cardiólogos quienes estamos involucrados en esta trascendental misión de actuar ante este flagelo, de ahí que el aprendizaje y la educación médica sean los pivotes fundamentales de nuestro oficio.

Como recordarán, una de las propuestas de plancha hace un año fue la de incrementar la educación médica continuada, y más importante aún, propender para hacer accesible el conocimiento de nuestra especialidad a quienes les puede ser muy útil, esto es, a los médicos de atención primaria, quienes inicialmente son los que enfrentan el primer contacto con el sujeto en riesgo o con el enfermo cardiovascular.

Es una verdad absoluta que los cardiólogos estamos llegando tarde. Iniciamos nuestro trabajo ante un desenlace o en el mejor de los casos en pacientes con comorbilidades en quienes ya existe un daño vascular y/o cardiaco. Esto es, la etapa 3 de la enfermedad.

Muchas veces empezamos a actuar porque el enfermo, por ignorancia o por no recibir un manejo oportuno $\mathrm{y} / \mathrm{o}$ adecuado, o por ser remitido de manera tardía, llega a un servicio de urgencias para que se le realicen procedimientos de intervencionismo. En razón de lo anterior, se propuso el "Primer congreso colombiano de Cardiología para médicos de atención primaria".

Este se ha centrado primordialmente en los temas del quehacer diario cardiológico, esto es: síntomas cardiacos y manejo integral de los factores de riesgo.

En cuanto síntomas cardiacos la temática se dirige al diagnóstico diferencial, qué hacer, qué no hacer y cuándo remitir, y en lo referente a factores de riesgo, definición del problema, qué hacer, qué no hacer, cuándo remitir y cómo prevenir.
Cada capítulo y grupo de trabajo cumplió el mandato de incluir dentro de su especialidad, un tema para atención primaria.

Es así, además, como hoy recibimos médicos internistas, familiaristas, urgenciólogos, intensivistas y generalistas. ¡Bienvenidos!

Seguiremos con nuestro compromiso de educación médica continuada a través de la página web mediante cursos virtuales y webinars, aclarando dudas con los expertos, y otras actividades; adicionalmente, están en desarrollo apps prácticas y en forma de algoritmo en dispositivos para que estén al alcance de los médicos de atención primaria.

Se tiene pensado institucionalizar el "Congreso para Atención Primaria" como actividad independiente y no unida a esta labor titánica de tres congresos en uno.

Y, en referencia a labor titánica, felicito al Dr. Mauricio Duque, quien organizó con el Capítulo de Electrofisiología y el Colegio Colombiano de Electrofisiología, el "Simposio internacional de arritmias", que ofreció una programación de lujo y fue dividido en tres secciones simultáneas: Electrofisiología para electrofisiólogos, Electrofisiología para el cardiólogo clínico y Electrofisiología para el médico no cardiólogo, más un exuberante plus: un curso de Electrocardiografía avalado por la Sociedada Colombiana de Cardiología y el CES, de un día de duración y sin costo adicional.

El simple pero crucial hecho de manejar nosotros los cardiólogos tratemos las patologías que ocasionan más morbi-mortalidad, alta discapacidad, impacto en los QUALY, y un alto costo, nos obliga a ser la Sociedad Científica de Colombia, líder en educación médica. Y así se hará y se cumplirá, e igualmente se les informará el logro de la meta dentro de un año cuando en esta misma asamblea y ante ustedes, se entreguen las banderas o la cosecha de lo que desde el inicio ha sido nuestra misión, el qué y el porqué.

El cómo, desde hoy, se compartirá con cada uno que se interese o se motive por este compromiso y nuevo camino. Los medios y los contactos, ya los tenemos. De modo que todo aquel que se sienta orgulloso de ser miembro de la Sociedad Colombiana de Cardiología y Cirugía Cardiovascular, es bienvenido a ayudar, bienvenido a trabajar.

Comienza ahora una nueva era de la Sociedad y una nueva actitud frente los recientes cambios en nuestro sistema de salud.

Indudablemente el médico generalista queda empoderado para no ser simplemente un contenedor del gasto o un remitidor, sino un actor crucial dentro del proceso de integralidad de la salud, y ya como derecho fundamental, según la Corte, pero con responsabilidad y racionalidad en el gasto.

Esto obliga precisamente a una educación médica concreta, práctica y de amplia cobertura. Para tal fin hemos se han firmado alianzas estratégicas con el Ministerio de Salud y Protección Social, con la Asociación colombiana de empresas de medicina integral -ACEMI-y otros entes de salud.

Con relación a educación médica, vale la pena destacar que se tienen más facultades de medicina que todos los EEUU (59) - hasta anteayer que se hizo la averiguación -, pero muchas, tienen una formación deficiente en el concepto de Salud pública, Semiología, Fisiopatología (ahora ya con categoría de plausibilidad), terapéutica y conocimiento 
y aplicación de guías, al igual que una gran falencia en la interpretación crítica de la literatura científica, y más grave aún, el desconocimiento de la Deontología médica: el deber del médico ante su paciente.

La nueva visión de las sociedades científicas deberá ser complementaria o suplir estas carencias.

Por desgracia, las sagradas condiciones de interrelaciones basadas en la confianza por parte del enfermo, y de tiempo y bondad por parte del médico, de la hasta hace poco estrecha relación médico-paciente, paso a ser intermediada, cronometrada y mal remunerada, añadiéndosele la carga administrativa de diligenciamiento de formularios, clasificación única de procedimientos en salud -CUP-, registro individual de prestacionbes de salud -RIP-, etc.

Esto acorta más ese valioso tiempo, durante el cual el médico dedica muchísimos más minutos a ver la pantalla del computador que la cara y el cuerpo expuesto del enfermo para un examen físico completo.

Pero, aparentemente el problema parece no ser de ahora, ya en el siglo XIx Samuel Langhorne Clemens, más conocido como Marck Twain, decía: "Los médicos son personas que formulan medicamentos de los cuales conocen poco, para enfermedades que conocen menos, a enfermos que casi no conocen' .

Yo, en nombre de la unanimidad de la junta directiva, comunico a Ustedes que seguiremos comprometidos con máximo esfuerzo, en la educación médica continuada, nuestra real razón de ser, misión y visión.

En este segundo año de Junta se rediseñará la página web, nuestro verdadero portal hacia la educación, en compañía de alianzas que ya se han firmado con otras sociedades científicas de la región, para intercambiar, aunar y compartir esfuerzos e ideas.

No sería la educación médica algo perfecta si no involucramos y nos dirigimos a la comunidad. Para tal fin, se está reacordando con la Fundación colombiana del corazón, de la Sociedad Colombiana de Cardiología y Cirugía Cardiovascular -cuya labor ha sido encomiable e incesante-, la ampliación de nuestro campo de acción hacia la prevención y promoción masiva y en temas puntuales, que tengan impacto en salud pública y que puedan ser medibles, tales como cardiología de la mujer -la ignorancia que mata-, ataque cerebrovascular e hipertensión arterial, fibrilación atrial y cardioembolia, diabetes y daño panvascular, y adolescencia, además de jornadas de detección de hipertensión arterial, fibrilación atrial y dislipidemias, con el terreno ganado, ya que tenemos apoyo y un acuerdo marco firmado por parte del Ministerio de salud y protección social, ACEMI y, como se mencionó, otros entes de salud.
Todos conocen que cada vez es más difícil ejecutar un evento en salud, ya que la industria farmacéutica, que realmente está comprometida con la educación médica, está cada vez más constreñida. A los del negocio de genéricos solo les interesa eso, el negocio, y no la inversión en educación médica ni en investigación, y muchas veces tampoco les importa la calidad -ya lo hemos vivido cotidianamente. Sin embargo, se pudo financiar este congreso, que aunque con menos participantes y conferencistas patrocinados por la industria, por primera vez suman 200 profesores, todos de altísima calidad, 221 conferencias, y lo más relevante, entre los nacionales están todos aquellos que a través de los Capítulos y los recién creados Grupos de Trabajo, respondieron trabajando por y con la Sociedad Colombiana de Cardiología y Cirugía Cardiovascular. Y es que nuestra Sociedad no la constituye la Junta y su nómina selecta de empleados; verdaderamente la constituimos todos los que la amemos y la sintamos. El deber de la Junta directiva no es solo realizar un congreso anual y apoyar incondicionalmente nuestro órgano de difusión y también puerta hacia el impacto por sus contenidos: nuestra Revista de Cardiología. Nuestros desafíos van más allá, y hacia allá llegaremos.

Finalmente, sin el compromiso, empeño y entusiasmo de nuestros empleados y contratistas, créanme que hubiese sido imposible que estemos bajo este recinto, aprendiendo y enseñando, agradeciendo y haciendo reconocimiento a aquellos que han dedicado toda su vida a la cardiología colombiana y a sus enfermos; que nos enseñaron, y poder además interactuar con los dirigentes gubernamentales en las políticas y estrategas de la salud, pero quizás lo más importante, encontrarnos o reencontrarnos con los amigos que desde lo más profundo de nuestras fibras de Purkinje queremos, y con quienes aún compartimos este maravilloso viaje y milagro de la vida.

¡Bienvenidos!

Mil gracias por su tiempo y atención.

\section{Enrique Melgarejo Rojas}

\section{Presidente Sociedad Colombiana de Cardiología y Cirugía Cardiovascular Correo electrónico: enrique.melgarejo@gmail.com}

http://dx.doi.org/10.1016/j.rccar.2017.05.003 0120-5633/

(C) 2017 Publicado por Elsevier España, S.L.U. en nombre de Sociedad Colombiana de Cardiología y Cirugía Cardiovascular. Este es un artículo Open Access bajo la licencia CC BY-NC-ND (http:// creativecommons.org/licenses/by-nc-nd/4.0/). 